\title{
LA CUARTA VOZ: LA IDEA DE COLECTIVO COMO INTERLOCUTOR
}

\author{
Verónica Soria Martínez \\ Investigadora independiente. Cràter (Col·lectiu de Recerca en Art, Teoria i Educació) \\ Neus Lozano Sanfèlix \\ Universitat Jaume I Castelló. Dep. d'Educació - Didàctica de l'Expressió Plàstica. Cràter (Col/lectiu \\ de Recerca en Art, Teoria i Educació)
}

\section{Resumen}

La construcción de la identidad del artista viene íntimamente ligada a la idea de práctica artística. En el estudio que presentamos a continuación, pretendemos identificar, dar visibilidad y cuestionar los discursos patriarcales (y neoliberales) que hoy en día siguen vigentes en las prácticas artísticas contemporáneas y que de manera indisociable repercuten en la construcción de nuestra subjetividad. Como agentes y participantes de esta investigación, se han analizado como caso de estudio las dinámicas organizativas y los obstáculos encontrados en el funcionamiento del colectivo Cràter, al que pertenecemos las autoras del texto y que fue fundado con la idea de construir un espacio protegido de reflexión y acción totalmente independiente de las dinámicas institucionales de la academia y las exigencias del tardocapitalismo. Cràter no solo es, sino que también se define, como un colectivo de mujeres, feministas, con vocación y formación artística, que busca nuevos modos de entender y hacer en arte, su investigación y su enseñanza.

\section{Palabras clave: FEMINISMO; COLECTIVO; METODOLOGÍA; INVESTIGA- CIÓN ARTÍSTICA}

\section{THE FOURTH VOICE: THE IDEA OF THE COLLECTIVE AS AN INTERLOCUTOR}

Abstract

The construction of the artist's identity becomes closely tied to the idea of artistic practice. In the following study, we aim to identify, to give visibility, and to question the patriarchal (and neoliberal) discourses that continue to be prevalent in contemporary art practices and that inevitably affect the construction of our subjectivity. As agents and participants in this research, we have analyzed as a case study the organizational dynamics and the obstacles we find in the functioning of the Cràter collective, to which the three authors of this text belong and that was founded with the idea of building a protected space for reflection and action, as one totally independent from the institutional dynamics of academia and the demands of late capitalism. Cràter is not just a collective formed by feminist women that have an artistic vocation and formation, but defines itself as such, looking for new ways of doing in the practice of art, research, and education.

\section{Keywords: FEMINISM; COLLECTIVE; METHODOLOGY; ARTISTIC RESEARCH}

\footnotetext{
Soria Martínez, Verónica y Lozano Sanfèlix, Neus 2017 "La cuarta voz: La idea de colectivo como interlocutor". AusArt 5 (1): 25-37 D0I: 10.1387 /ausart.17788
}

\section{AUSART}




\section{INTRODUCCIÓN}

Generar un espacio de pensamiento (Sheikh 2009), como forma de trabajo compartido, nos obliga a replantearnos muchas de las ideas que asociamos a los modos de hacer en la producción artística y académica. Cràter nace precisamente a finales del 2013 con esta vocación, la de establecer un espacio de convivencia para el pensamiento y la acción que, más allà de la función de empoderarnos como colectivo, nos permitiera construirnos un marco referencial común que nos sirviera para cuestionar el relato del artista y del arte que hemos heredado. $Y$ de esta manera, adoptar una postura emancipada desde la que posicionarnos y trabajar conjuntamente como mujeres artistas. Nuestro objetivo no era tanto el de reivindicar un espacio dentro del arte, sino el hecho de ofrecernos a nosotras mismas, la oportunidad de imaginar otras identidades para la artista y su práctica, puesto que parte de los interrogantes que nos planteamos surgen en gran medida de las contradicciones que se generan al abordar nuestro posicionamiento como artistas y como mujeres en una sociedad patriarcal y neoliberal. Conscientes de que la idea de colectivo siempre ha estado presente en el momento en que se han producido giros en la producción y la recepción del arte, otro de nuestros objetivos también ha sido el de dotarnos de acciones que nos permitan aproximarnos a este cambio, que consideramos cada vez más necesario. A continuación, expondremos cuáles han sido las dinámicas organizativas y los obstáculos que nos hemos encontrados como parte de este camino que apenas estamos empezando a andar y que cada vez estamos más convencidas que a lo que nos conduce, es a un giro o cambio en el que lo que se descentra principalmente es la figura individualista del artista que aún hoy sigue vigente. En este sentido, este texto es una manera de repensarnos y de adquirir un compromiso de cara al futuro próximo y que pensamos que puede ser de interés como intercambio de ideas.

\section{Punto dE PARTIDA}

Al plantearnos interrogantes feministas en producción artística, decidimos poner a examen nuestra práctica, nuestra razón de ser y nuestros objetivos. En primer lugar, porque pretendemos que nuestra práctica sea consecuente con nuestras ideas, y también porque parte de nuestra dinámica consiste en poner todas las ideas en continuo cuestionamiento, comenzando por nosotras mismas. Esto originó que tuviésemos que enfrentar ciertos núcleos de conflicto que podríamos identificar con dos contextos diferenciados: el que atañe a la esfera artística, alrededor de ideas que construyen la subjetividad de lo que entendemos por arte y artista; y el profesional/personal, que deviene de 
ser mujeres. Estos dos campos, no sólo inciden de manera directa, sino que también se interrelacionan creando sinergias mucho más complejas.

En lo que se refiere a la construcción de la subjetividad del artista encontramos los siguientes "conflictos":

- La imposibilidad de conciliar la vida familiar con la laboral.

- El sometimiento a dinámicas de trabajo, organización y priorización de tareas según estándares de eficiencia, productividad y comerciabilidad.

- La presión para incrementar la producción, lo que lleva a en muchas ocasiones desistir y dejar de lado aquellos proyectos que no se traducen en un reconocimiento profesional.

- La precariedad que conduce a una situación apremiante y que sitúa a los individuos en una posición de competitividad constante

En cuanto a las condiciones reales del estatus de la mujer en nuestra sociedad, encontramos los siguientes obstáculos:

- La desigualdad de género en el acceso a un empleo digno y estable.

- Dificultades particulares derivadas de la imposibilidad de conciliar la vida familiar con la laboral.

- Una inseguridad aprendida socialmente (este aspecto se desarrolla ampliamente en Solnit 2014).

A continuación trataremos de analizar estos obstáculos y cómo hemos decidido trabajar con ellos, buscando la manera de neutralizarlos para trazar posibles líneas de fuga, de trabajo futuro, y en definitiva, de repensar cómo sería ese otro arte.

\section{DESEOS y EXPECTATIVAS}

La formación de Cràter es una respuesta a los conflictos y obstáculos encontrados en torno a los dos contextos mencionados anteriormente:

Respecto a los conflictos generados en torno a la construcción de la subjetividad del artista, Cràter pretende ser un colectivo artístico que se forma con la perspectiva de cambiar el paradigma de lo artístico. Frente a una estructura de 
mercado que comercializa el objeto artístico y objetifica al artista, concebimos la tarea del artista como una actividad social, compartida, disidente y libre, que permite reestructurar la realidad cotidiana para de esta manera repensar la organización y percepción de las convenciones sociales existentes. El arte basado en el objeto y en la voz individual del artista no nos parece tan interesante como la creación de situaciones y experiencias compartidas.

En cuanto a los obstáculos producidos por las condiciones reales del estatus de la mujer en nuestra sociedad, Cràter quiere convertirse por un lado en un apoyo mutuo y por otro en un cuestionamiento de las lógicas patriarcales y neoliberales en el ámbito de la producción del conocimiento. El apoyo lo conseguimos compartiendo conocimientos, aprendizajes y experiencias, mientras que el cuestionamiento y la producción de conocimiento se genera tanto mediante el producto de nuestro trabajo como en las posibles maneras de desarrollarlo.

Para ello, la forma que toma el colectivo es fundamental, puesto que sitúa a sus componentes como interlocutores unos de otros. Mediante la interacción entre ellos, se generan más ideas, que se someten a una mayor profundidad de análisis y dan lugar a proyectos más ricos. En la reflexión que se genera a partir de nuestra práctica artística, nos parece importante la creación de una voz común, la del colectivo, no ya como suma o consenso de las partes, sino como evolución de las posiciones a través de un proceso de cuestionamiento mutuo. De ahí el título de este texto.

\section{MÉtodo y PRÁctica}

Nos interesa emplear metodologías artísticas en las prácticas institucionales. En otras palabras, activar maneras de funcionar que nos permitan participar de las instituciones, pero tratando de desplazar desde abajo los límites de lo que se permite y se promueve desde arriba. A continuación describimos los métodos que orientan nuestra práctica artística y cómo se relacionan con ella. Aunque nuestro trabajo se encuentra todavía en sus inicios y algunos de los ejemplos descritos aún están siendo desarrollados, nos sirven para delinear modos de funcionamiento y establecer aprendizajes para el futuro. Estos son los pasos que hemos emprendido para neutralizar obstáculos y núcleos de conflicto desde el género, la práctica artística y la academia.

Creación de un intersticio de funcionamiento cooperativo en el que podamos actuar de manera libre y ejercer la disidencia, dentro del colectivo, esto 
es, tratando de operar al margen de las lógicas de funcionamiento propias del neoliberalismo, y fuera del colectivo, buscando la manera de desplazar estas lógicas en nuestras prácticas sociales. Para ello, se requiere un aprendizaje que consiste en desaprender los hábitos que asimilamos durante nuestros años de formación. Por ejemplo, en la educación básica se nos enseña a reproducir contenidos aprendidos y asumir el rol de emisor o receptor de conocimiento. Sin embargo, hemos comprobado que es gracias a la colaboración y al debate que se construye un pensamiento divergente para generar un discurso crítico.

Cuando en 2014 concebimos la realización del taller "Derives entorn a la ciutat. La natura com a resistència", en el marco de la iniciativa "Parlem d'art públic", impulsado por la Universitat de València y que tendría lugar en una galería de arte situada en el barrio de Russafa (València), decidimos centrarnos en las sinergias que actúan en el barrio, en proceso de gentrificación. Para ello, invitamos a otras tres personas: Carles Dolç, un urbanista especializado en los cambios en la ciudad debidos a movimientos sociales; Mariví Martín, una activista de la asociación de vecinos del barrio, que nos contó las luchas y las fuerzas presentes en el barrio; y un botànico, Simón Fos, que realizó una guía por las especies vegetales autóctonas que crecen en el barrio a pesar de la fuerte urbanización. Así, en lugar de una conferencia-taller, se generó una práctica artística alrededor de la deriva y la figura del flâneur, en la que se invitaba al público asistente a participar del paseo y del debate.
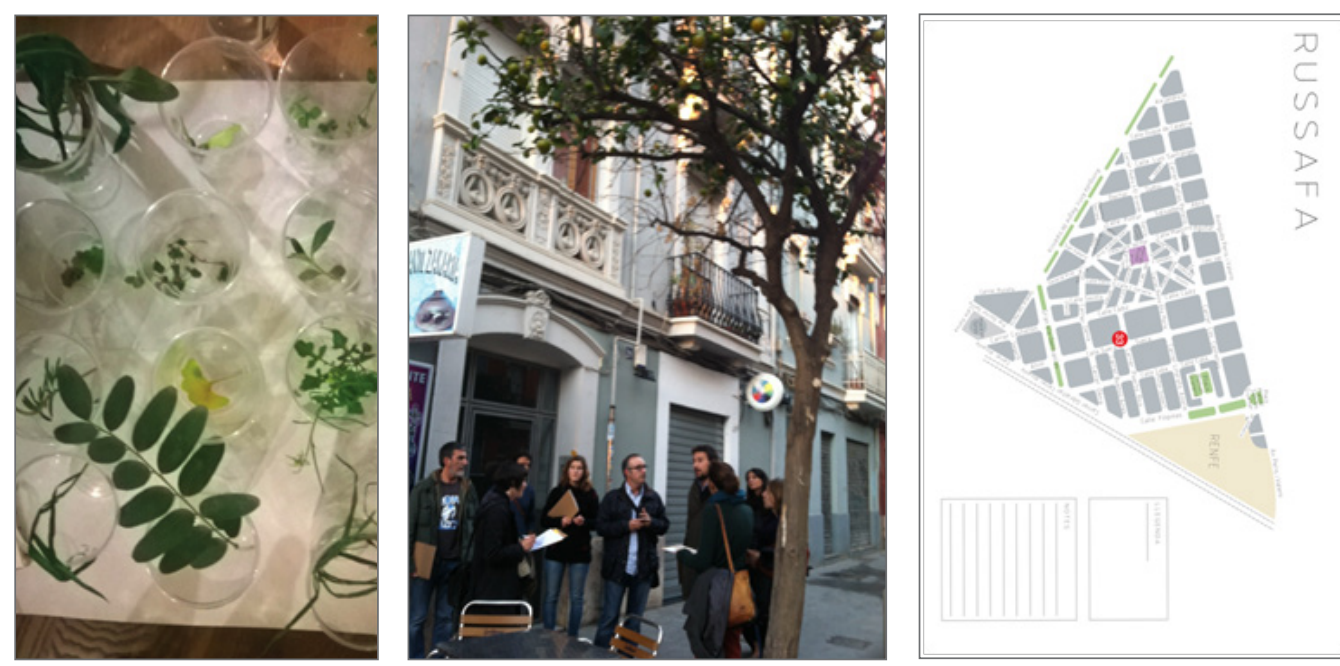

Derives al voltant de la ciutat. La natura com a resistència, Cràter, taller experimental, 2014. 
Voluntad de cuestionamiento continuo como brújula de pensamiento. Esto tiene por objetivo preservar nuestra coherencia tanto en la forma como en los contenidos. Por ejemplo, a menudo abordamos una idea que se presenta atractiva, pero que no obstante, tras un detenido análisis, y expuestas las diferentes perspectivas del colectivo, acertamos a detectar las contradicciones que entran en conflicto con nuestros deseos y expectativas. Debido a la internalización de ideas dominantes promovidas por el lenguaje y el pensamiento neoliberal, lo que trabajando en solitario podríamos no advertir, el trabajo cooperativo nos permite estar alerta a las consecuencias de lo que producimos, al ponerlo en común con puntos de vista divergentes. El producto resultante de este modo de colaboración es mucho más rico e interesante, puesto que es el resultado de un debate continuo, en el que intervienen los diferentes bagajes de cada una. Pero naturalmente, trabajar de este modo no es fácil ni rápido, por lo que resulta muy poco prolífico o productivo en términos de producción comercial: nuestra búsqueda no se sustenta en la obtención de unos resultados, sino en la experimentación del proceso, en el cómputo de cuestiones que surgen y multiplican los significados.

Esta voluntad puede detectarse en el texto que realizamos cuestionando nuestra obra Mapa dels desitjos, "Arte y educación: espacios comunes" (Vidagañ, Soria \& Lozano 2016), así como en el presente texto, por el proceso que hemos seguido al escribirlo. En ambos hemos puesto nuestra experiencia en perspectiva. Si bien en el primero pudimos extraer conclusiones a partir de las múltiples interacciones y al realizar, escuchar y emplazar las entrevistas, y contrastarlas con nuestros objetivos y resultados, en este texto se trata más bien de nuestra metodología la que hemos decidido examinar.

Este texto partió de la planificación de unas pautas que nos permitían tanto individual como colectivamente detenernos en el proceso de generación de ideas. Establecimos tres cuestiones que si bien no se vinculan entre ellas, si que convergen en cada una de nosotras y a su vez, conforman el colectivo: a) ¿Qué entendemos por arte? b) ¿Cómo nos pensamos, nos definimos? c) ¿Cómo imaginamos Cràter? Basándonos en estas preguntas escribimos tres textos cada una que subimos a nuestro blog. Los textos que escribió cada una fueron respondidos, comentados y cuestionados por las tres. Estas pautas nos sirvieron para establecer una base y primera aproximación a nuestra identidad tanto individual como colectiva de manera no-lineal. De este modo la construcción del texto no asumía una direccionalidad definida, pregunta-respuesta o objetivo-metodología, sino que se convertía en una práctica especulativa experimental, que además no asumía la estructura y la lógica de un texto 
propiamente académico. El proceso abarcaba tanto la experiencia individual como el posicionamiento consensuado como colectivo. Con un formato más de collage que de narración, tomando más una forma estructural de ensayo visual que de narración discursiva.

Al mismo tiempo, en nuestras reuniones semanales discutíamos verbalmente las mismas cuestiones y tomábamos apuntes, que también eran subidos al blog. A partir de este debate se comenzó un documento en línea que ya contenía las diferentes aportaciones que habíamos ido generando y recopilando. Durante el procesamiento de este documento se ha conseguido una producción consensuada mediante el uso de las herramientas de comentarios, la edición de texto en modo sugerencia, el chat y la videoconferencia simultáneamente. De esta manera podíamos escribir, bien mientras discutíamos al mismo tiempo, o bien editar y sugerir sobre el trabajo inmediatamente anterior cuando no podíamos coincidir.

Lo importante al respecto no es el uso en sí de estas herramientas, sino la aproximación fragmentada que permiten para elaborar un enfoque con diversas perspectivas que se interrelacionan simultáneamente. De la intersección de estas perspectivas, de las respuestas y preguntas, empezamos a constatar las conexiones entre lo privado y lo público, entre lo personal y lo político. Es a partir del debate generado que surge una cuarta voz.

Convergencia de tiempos. Si en la producción de conocimiento se premia el trabajo eficaz, especialmente con las últimas transformaciones que se están dando en la academia (Lozano Vidagañ, Soria \& Lozano 2016; Ordine 2013), nosotras proponemos desarrollar un modo de trabajo que sea verdaderamente reflexivo aunque lento, deliberadamente ineficiente, en el que tengamos tiempo para cuestionar cada idea.

Nuestra pieza Texto hiperlocal, que se encuentra en fase de producción y que será presentada en el marco del festival urbano Intramurs, presenta una pieza de realidad aumentada en el espacio público, en la que el viandante puede escanear códigos QR situados estratégicamente en el centro histórico de la ciudad que dan acceso a una variedad de materiales, entre los que se pueden encontrar noticias, recuerdos, fragmentos de literatura, mini episodios audio y fragmentos de poesía visual relacionados con su ubicación geográfica. Esto abre un plano de cohabitación de diversos espacios temporales que convergen en una misma pieza y que se relacionan con el espacio público. 
La idea original para este proyecto ha ido evolucionando a través del tiempo, a partir de protoideas generadas y compartidas en línea y que hemos ido madurando en diversos lapsos temporales de frecuencia irregular. En un primer momento, el proyecto no incluía la variedad de materiales ni el componente de realidad aumentada, sino que fuimos añadiendo estos elementos poco a poco conforme íbamos discutiéndolo en el blog. En este sentido, el blog nos permite ir añadiendo cuestiones que quedan registradas para un momento posterior, dando la oportunidad de asentar el pensamiento y permitir su transformación a través de diferentes sugerencias y críticas. Esto contrasta con la inmediatez de las reuniones, aportando otro tipo de generación de ideas más reposado.

Convergencia de espacios. La red. Para superar el inconveniente de la distancia (vivimos en diferentes continentes), nuestras reuniones deben ser mediante videoconferencia y chat. Aunque la comunicación se ve interrumpida y a menudo interferida por el medio y por los malentendidos que provoca, esta manera de trabajar ha facilitado la creación de un archivo común en línea, a partir de las carpetas y documentos compartidos en red. Por otro lado, al trabajar en el blog como herramienta, subiendo videos, discusiones, referencias y propuestas para proyectos, se desarrolla una especie de línea temporal hipertextual e hipermedia de cada proyecto. En este sentido, hemos superado lo que era inicialmente un obstáculo para posibilitar la construcción de una discusión en progreso constante. Esto nos ha permitido compartir aprendizajes sobre nuestros métodos de trabajo, sobre nosotras mismas y sobre nuestra manera de trabajar colectivamente; $y$ ha demostrado la importancia de respetar los tiempos y procesos de cada una.

Esta forma de trabajo alrededor del espacio físico y el espacio red queda reflejada en el modo operante y en el resultado de nuestra pieza Mapa dels desitjos. Voces de la comunidad universitaria. Esta intervención en el espacio público, presentada con motivo de la XVII Mostra d'art públic per a joves creadors en octubre de 2014, incluye un archivo de entrevistas sobre los deseos de los diversos miembros de la universidad, así como un mapa sonoro online del campus, con las entrevistas ubicadas en los lugares preferidos de los entrevistados. Nos parecía interesante plantear cómo el espacio educativo se interrelaciona con los deseos y ambiciones de estudiantes, profesores y trabajadores de la universidad. Esta reflexión sobre el espacio urbano y sus extensiones via realidad aumentada ha servido para definir el mencionado anteriormente Texto hiperlocal. 


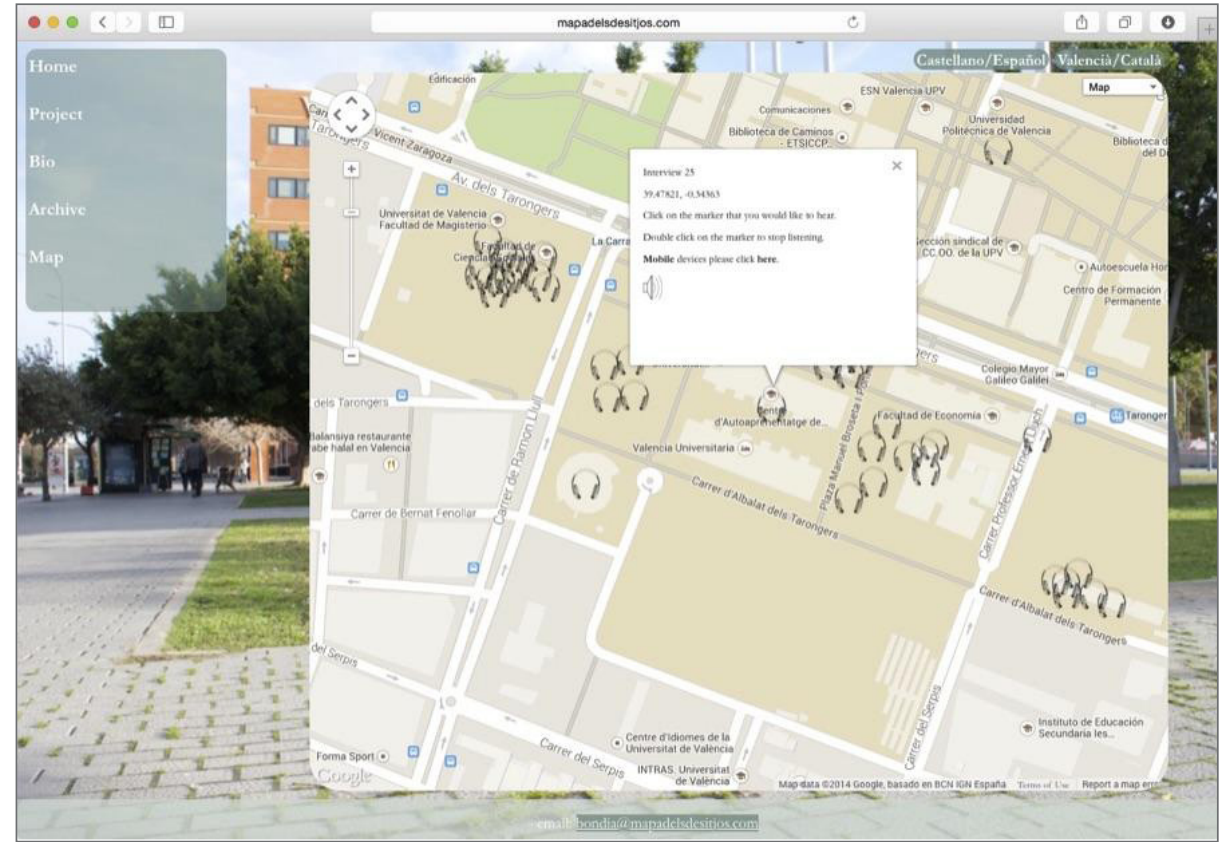

Mapa dels desitjos, Cràter, plataforma interactiva online, 2014.

Cuidado de las relaciones interpersonales dentro del colectivo. El continuo cuestionamiento y la falta de una cultura verdaderamente crítica puede hacer que se generen sentimientos negativos al rebatir nuestras ideas. Aunque esto puede relacionarse con el ego, que afecta a ambos géneros, a las mujeres se nos educa tradicionalmente para conciliar, para estar de acuerdo y para gustar (Adichie 2015). Debatir las ideas de nuestras compañeras nos genera cierta incomodidad inicial. Por ello se vuelve especialmente importante utilizar un lenguaje reparador.

Por otro lado, el trabajo colectivo es imposible disociar del trabajo individual, y esto crea conflictos como el que las personas que forman el colectivo no sientan debidamente reconocidas sus aportaciones. Esto tiene mucho que ver con el capitalismo cognitivo y la dificultad de poner horarios y límites al pensamiento, así como de cuantificar el valor de los conocimientos. El producto del así llamado trabajo creativo es intangible, y solo se hace visible cuando se aprovecha para extraer un rédito económico (de manera directa o indirectamente, "haciendo currículum" para poder ascender económicamente). En este sentido, de nuevo el lenguaje que usamos cobra especial importancia, y se requiere una conciencia del trabajo común como ente colectivo. 
Generación de apoyos frente a la competitividad desenfrenada. Una de las estrategias de la economía neoliberal es hacer al trabajador competir con sus iguales. En el caso de la trabajadora, se le genera además una manera especial de inseguridad, pues se le compara constantemente con un ideal de perfección (dentro y fuera del entorno laboral) imposible de conseguir. Por tanto, los conflictos que se derivan de la competitividad y la inseguridad son una consecuencia de la precariedad y la ideología del trabajo en una sociedad capitalista. Una característica de nuestra voluntad de cuestionamiento continuo es recordar que el sentimiento de inseguridad y la necesidad de competir vienen dados por nuestra situación social, por lo que no deberían tener cabida en nuestro modo de funcionamiento. Sin embargo, es clave aceptar que emergen en nosotros debido a nuestro funcionamiento en sociedad. De nuevo, rebatir no a la persona, sino a los obstáculos y contradicciones que nos vienen impuestos al querer funcionar de manera diferente a las estructuras de empresa.

El proyecto Nunca te acostarás sin aprender una cosa más, en fase de producción, tenía como objetivo detectar los aprendizajes que adquirimos diariamente y que en la mayoría de casos, pasan desapercibidos. El proceso de investigación consistió en descubrir

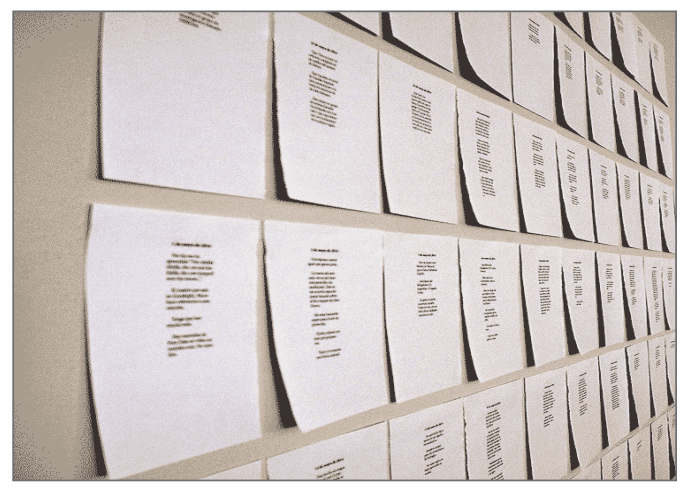

No te acostarás sin saber un cosa más, Cràter, papel, 2014. y anotar día a día todo aquello que podía formar parte de un nuevo aprendizaje. Ello permitió visibilizar aspectos de nuestro pensamiento que a menudo son invisibles, y compartirlos. Al poner esas anotaciones ordenadas por días, ayudó a observar la correlación en el tiempo de estos registros, cuya única coincidencia era el día. Esto fue un ejercicio en comprensión mutua de nuestros procesos cognitivos que tratamos de incorporar a nuestro funcionamiento cotidiano.

Compilación de saberes realmente útiles del aquí y ahora, como colectivo artístico de mujeres por un cambio en el paradigma de lo artístico. Lo que realmente necesitamos es otro arte, diferente del arte objeto. En este sentido, lo que necesitamos saber no es cómo entrar en los procesos burocráticos académicos, sino cuáles son las acciones que podemos emprender para bloquear el sistema. En nuestro texto 
"La formación artística universitaria como interfaz" (Vidagañ, Soria \& Lozano, 2016), apuntamos a cómo el docente, en tanto que un error de diseño del sistema, puede generar una disrupción en la eficiencia educativa que genera estudiantes-producto. Este es un concepto que continuamos desarrollando. De nuevo el tipo de lenguaje que usa el docente-bug es crucial para generar este cambio positivo en su entorno.

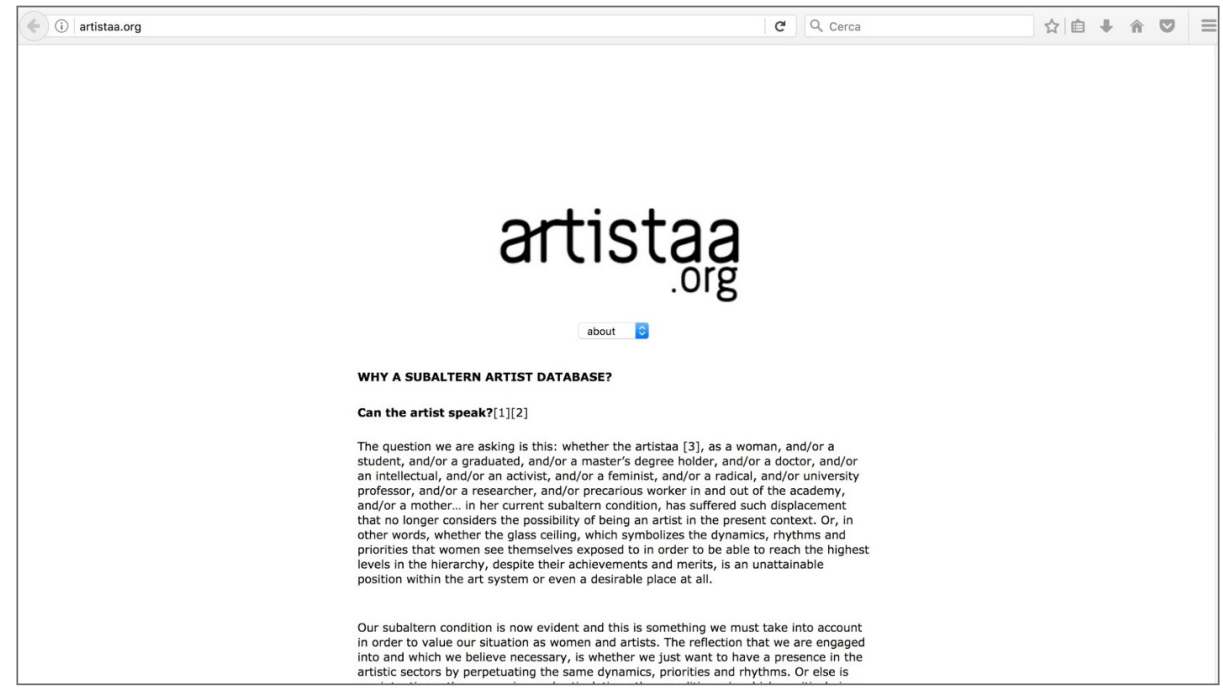

artistaa.org, Cràter, base de datos online, 2017.

Una acción que hemos emprendido al respecto, orientado hacia ese cambio de paradigma en el arte que mencionamos al inicio, es la creación de la base de datos artistaa.org. En ella, recopilamos los datos de aquellas que quieran participar, para podernos hallar y referenciar, como un directorio artístico y conceptual. Primero, presentamos el concepto de artistaa, término prestado de Silvia Martí Marí (2016) para designar a la mujer artista, y que nosotras extendemos a las artistas conscientes de su condición de subalterna en el mundo del arte como movimiento especulativo, y que como tales rechazan participar de él en los términos prescritos tradicionalmente. Las artistaas pueden registrarse enviando sus señas, su web y una foto o texto con que quieran presentarse en la base de datos. Esto nos ayuda como investigadoras para poder hallar otros referentes diferentes de aquellos apoyados por la historia oficial y por el arte de mercado. 


\section{Conclusiones}

Consideramos que la potencialidad de lo colectivo no radica solo en una mirada consensuada sobre determinada idea. Mientras cada uno de los miembros del colectivo, con su bagaje y sus experiencias comparten sus modos de pensar y hacer, de este diálogo surge una posición nueva, que de manera recíproca se convierte en interlocutora, la voz colectiva. A pesar de la lentitud de los procesos reflexivos y de los conflictos (internos y con el resto de la sociedad), nuestra conclusión es que no solo es muy fructífero sobrepasar este obstáculo inicial y analizar los diversos aprendizajes y construcciones que pueden derivarse de la colaboración, sino que además es necesario ejercerla de esta manera, a modo de resistencia y reivindicación para advertir de la existencia de otras vías, que son posibles y deseables.

El hecho de que en el actual contexto capitalista y neoliberal, no se favorezcan las estructuras colectivas, especialmente si no son jerárquicas, es un síntoma de la ideología implícita en los procesos que que estructuran nuestra sociedad, que fomenta la individualidad e intercede en las dinámicas no individualistas. Por este motivo, se desfavorecen este tipo de asociaciones, haciendo que se rompan los vínculos individuales de los componentes del colectivo. El ámbito académico está asumiendo estas sinergias, afectando la producción de conocimiento en su detrimento. Solo creando dinámicas no jerárquicas que no potencien el individualismo, podremos encontrar las estrategias necesarias para combatir estos modos de hacer.

\section{Referencias}

Adichie, Chimamanda Ngozi. 2015. We should all be feminists. 23. First presented as a TED Talk (2012). London: Four Estate

Asensi Pérez, Manuel. 2009. "La subalternidad borrosa: Un poco más de debate en torno a los subalternos". En ¿Pueden hablar los subalternos?, Gayatri Chakravorty, trad. y ed. crítica de Manuel Asensi Pérez. Barcelona: MACBA

Azadi, Sohelia. 2017. "Interview Sohelia Azadi” Cultural Reproducers (blog), 12 abril. www. culturalreproducers.org/2017/04/interview-soheila-azadi.html

Chadwick, Whitney. 1992. Mujer, arte y sociedad.Traducción, María Barberán. Barcelona: Destino

González Diéguez, Horacio. 2016. “¿Quién mató a Escoitar?”. VHPlab Make easy_(blog), 12 abril. 
Kaufmann, Therese. 2011. "Arte y conocimiento: rudimentos para una perspectiva decolonial". Traducción de Raúl Sánchez Cedillo Eipcp.net/Transversal, web Instituto Europeo para Políticas Culturales Progresivas. http://eipcp.net/transversal/0311/kaufmann/es/print

Lozano Sanfèlix, Neus. 2016. “¿Puede hablar la artista?”. En: Artistaas: Violencias, afectos, diálogos, creaciones, Silvia Martí Marí, ed. Zaragoza: Universidad de Zaragoza

Martí Marí, Silvia, ed. 2016. Artistaas: Violencias, afectos, diálogos, creaciones. Zaragoza: Universidad de Zaragoza

Offmother (Grupo de trabajo). 2016. "Pájaros en la cabeza". Laboralcentrodearte.org, web Laboral Centro de Arte y Creación Industrial www.laboralcentrodearte.org/es/actividades/ offmother

Ordine, Nuccio. 2013. L'utilitá dell'inutile: Manifesto. Con un saggio di Abraham Flexner. Milano: Bompiani

PIPES (Participatory investigation of Public Engaging Spaces). 2015. "Manifiesto para una aproximación crítica a la interfaz" Pipes.hangar.org, web oficial PIPES BCN, 12 abril. https:// interfacemanifesto.hangar.org/index.php/Main_Page/es\#Propuesta_de_acciones_relacionadas_con_los_puntos_del_manifesto

Sheik, Simon. 2009. "Objects of study of comodification of knowledge? Remarks on artistic research". Art\&Research 2(2): 1-8. www.artandresearch.org.uk/v2n2/pdfs/sheikh.pdf

Solnit, Rebeca. 2014. Men explain things to me. Chicago: Haymarket

Spivak, Gayatri Chakravorty 2002. “¿Puede hablar la subalterna?”. Traducido por $\mathrm{M}^{a}$ Rosario Martín Ruano. Asparkía Investigación Feminista 13: 207-14

Spivak, Gayatri Chakravorty. 1999. A Critique of postcolonial reason: Toward a history of the vanishing present. Cambrige, Mass: Harward University

Vidagany-Murgui, María, Neus Lozano Sanfèlix \& Verónica Soria Martínez. 2016. "La formación artística universitaria como interfaz". En Interface Politics. 1st International Conference, Teresa Martínez Figuerola \& Jorge Luis Marzo, eds., 377-94. Barcelona: GREDITS

Vidagañ Murgui, Maria, Verónica Soria Martínez \& Neus Lozano Sanfèlix. 2016. "Arte y Educación: Espacios comunes. Reflexiones en torno a la práctica artística 'Mapa dels Desitjos'”. EARI Educación Artística 7: 135-145. Doi: 10.7203/eari.7.8071

www.vhplab.net/spip.php?article324

(Artículo recibido 30-04-17; aceptado 09-06-2017) 